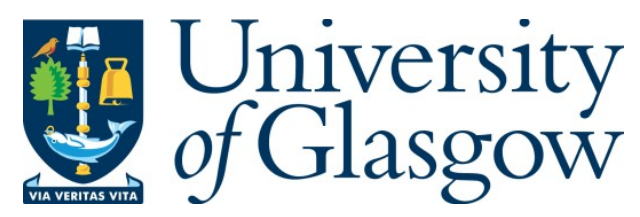

Navaratnarajah, S., Han, C., Dianati, M., and Imran, M. A. (2016) Adaptive stochastic radio access selection scheme for cellular-WLAN heterogeneous communication systems. IET Communications, 10(15), pp. 1986-1994.

There may be differences between this version and the published version. You are advised to consult the publisher's version if you wish to cite from it.

http://eprints.gla.ac.uk/132583/

Deposited on: 20 December 2016

Enlighten - Research publications by members of the University of Glasgow http://eprints.gla.ac.uk 


\title{
An Adaptive Stochastic Radio Access Selection Scheme for Cellular-WLAN Heterogeneous Communication Systems
}

\author{
Shobanraj Navaratnarajah, Chong Han, Mehrdad Dianati, and Muhammad Ali Imran \\ Institute for Communication Systems (ICS) \\ University of Surrey, Guildford, Surrey, GU2 7XH, UK. \\ Email: \{s.navaratnarajah, chong.han, m.dianati, m.imran\}@surrey.ac.uk
}

\begin{abstract}
This paper proposes a novel Adaptive Stochastic Radio Access Selection (ASRAS) scheme for mobile users in heterogeneous Cellular-WLAN systems. In this scheme, a mobile user located in dual coverage area randomly selects WLAN with probability of $\omega$ when there is a need for downloading a chunk of data. The value of $\omega$ is optimised according to the status of both networks in terms of network load and signal quality of both cellular and WLAN networks. An analytical model, based on Continuous Time Markov Chain (CTMC) is proposed to optimise the value of $\omega$ and compute the performance of proposed scheme in terms of energy efficiency, throughput, and call blocking probability. Both analytical and simulation results demonstrate the superiority of the proposed scheme compared to the mainstream network selection schemes, namely, WLAN-first and Load Balancing.
\end{abstract}

\section{Introduction}

The explosive growth of wireless data-traffic demand causes huge challenges for the cellular network operators to significantly increase the network capacity. One of the most promising solution for this challenge is the heterogeneous network (HetNet) architecture. A HetNet may consist of different size of cells with different radio access technologies (RATs), with overleaping coverage that complement each other [1]. The major advantage of such HetNet is the low power small-cells can increase the capacity of the network multi-fold, while introducing minimal deployment cost compared to the traditional macro-cells [2]. Hence, HetNets are expected to be one of the key aspect in the realization of the 5th Generation $(5 \mathrm{G})$ of wireless networks. In this regard, off-loading mobile data traffic to Wireless Local Area Network (WLAN) becomes increasingly popular among cellular network operators. This is due to the fact that the utilisation of unlicensed spectrum in WiFi brings additional bandwidth resources, instead of sharing the much scared and costly cellular frequency spectrum for small cells. Thus, cellular network operators already started to use WiFi to meet the capacity demands in their cellular networks [3]. This kind of operator deployed WiFi networks are sometimes referred to as Carrier-WiFi, where carrier class services can be expected [4].

Apart from increasing the network capacity, increasing the energy-efficiency (EE) of network also becomes an important challenge for the network operators, due to the increased cost on energy and environmental concerns. Studies have revealed that a majority of energy (50-80\%) is consumed in the wireless access part of mobile communication networks [2]. Hence, increasing the EE of access networks, has significant impact on total network EE. In this regard, the HetNet architecture is also regarded as a promising solution to improve the network EE. This is due to the fact that small 
cell access points (APs) can provide considerable capacity improvement, while consuming much less energy [2].

Although HetNet is considered as a promising component of the solution for both capacity and EE problems in future wireless communication networks, there are challenges related to effective operation of such networks, especially the integrated Cellular-WLAN HetNet. One of the major challenges is the optimal selection of the serving network [5]. It has been reported that the adopted network selection scheme has a considerable effect on the overall network performance [6]. Although there are some advanced network selection schemes in literature [7,8], the objectives of most existing schemes have been mainly limited to user perceived throughput and load balancing. Network EE has not been regarded as an important objective. Moreover, studies which focus on network selection in Cellular-WLAN HetNet, typically assume either a fixed system throughput regardless of channel condition or theoretical achievable throughput based on perceived Signal to Interference plus Noise Ratio (SINR). For example, in [9], it is assumed that WLAN network provides a fixed throughput of $7 \mathrm{Mbps}$ and cellular network provides $2 \mathrm{Mbps}$. On the other hand, in [10], the throughput of both WLAN and cellular networks are assumed to be equal to the maximum theoretical achievable throughput based on perceived SINR. However, in reality, the throughput of a practical network remains between these two extreme assumptions, mainly for two reasons. First, in the state-of-the-art wireless communication systems, adaptive Modulation and Coding Schemes (MCS) are used to enhance the spectral efficiency based on the channel quality. Thus, the throughput varies according to the channel quality. Secondly, technical limitations in real systems, such as limited number of MCS levels and signalling overheads caused by underlying multiple access schemes, prevent the system to achieve theoretical maximum throughput. Moreover, in a multi-RAT HetNet, different RATs may employ different physical (PHY) layer techniques and different multiple access schemes at medium access control (MAC) layer. The differences in MAC and PHY layer techniques have considerable impact on the users' perceived throughput as well as on the system performance of each RAT. Therefore, it is important to consider the realistic system throughput in order to optimise the network selection schemes, especially, for a multi-RAT HetNet. In addition, most of the works in literature that focus on Cellular-WLAN HetNet assume constant-bit-rate (CBR) services (e.g., voice services). For example, in [11], it is assumed that each user requires a fixed data-rate, in order to evaluate the call blocking and handoff failure probabilities. However, in the future wireless communication systems, major part of the traffic will be variable-bit-rate (VBR), sometimes referred to as elastic traffic (e.g., data traffic). To the best of our knowledge, there has not been any study that considers elastic traffic in Cellular-WLAN HetNet with adaptive MCS for both type of RATs.

Apart from the aforementioned shortcomings, most of the existing network selection schemes are user-centric, which imposes additional computational complexity to the limited hardware resources on the user terminals [7,8]. In addition, such user centric schemes add more signalling overhead to the network and the wireless links, in order to enable distributed network selection process. Therefore, in this paper, we propose a novel Adaptive Stochastic Radio Access Selection (ASRAS) scheme for data traffic in Cellular-WLAN HetNet, which aims to improve total network performance. In this scheme, a mobile user located in dual coverage area randomly selects WLAN with probability of $\omega$ when there is a need for downloading a chunk of data. Hence, the network selection can be stochastically controlled by optimising the value of $\omega$ according to the network load and channel quality. Since this is a network-centric scheme, the complexity is mostly limited to the network side. In addition, it allows the operators to influence the network selection decision by optimising the value of $\omega$ according to their objectives such as network EE, system throughput, 
and call blocking probability. The optimal value of $\omega$ is obtained by developing an analytical model based on Continuous Time Markov Chain (CTMC), which takes into account the system specific limitations (e.g., limited number of MCS and RAT specific overheads). The main contributions of this article are summarised as follows: 1) a novel radio access selection scheme (i.e., ASRAS) is proposed for data traffic in Cellular-WLAN HetNet that improves the total network performance; 2 ) in order to obtain the optimal value of $\omega$, and analytically evaluate the system performance, a comprehensive analytical model based on CTMC is developed; 3) comprehensive performance analysis is carried out to evaluate the performance of the proposed scheme and to compare with other mainstream network selection schemes.

The rest of the paper is organised as follows. A brief survey of related works on Cellular-WLAN HetNet is presented in Section 2. Section 3 presents the considered system model for the proposed scheme. The proposed ASRAS scheme is given in Section 4. An analytical model is developed to find the optimal value of $\omega$ in Section 5. In Section 6, the performance of the proposed scheme is evaluated and compared with the benchmark schemes. Finally, Section 7 concludes the paper.

\section{Related Works}

This section presents a brief survey of related works on Cellular-WLAN HetNet in the literature. Since off-loading of the mobile data traffic from cellular communication systems to WLAN gathered considerable momentum in recent years, the 3GPP standard Access Network Discovery and Selection Function (ANDSF), and the IEEE standard Media Independent Handover (MIH) were developed to facilitate the network selection and vertical handover in a multi-RAT environment $[12,13]$. Although the standards facilitate a framework for intelligent network selection, they do not specify how the network selection decision should be made. The decision making is left to the mobile devices. However, in general, network related policies are not considered by most of the devices. The devices make the network selection decision based on their local knowledge and the user preference. For instance, in the mainstream network selection scheme known as WLAN-first, the user equipment (UE) always tries to connect to WiFi, if there is a WiFi coverage available, regardless of the status of the WiFi network [14]. This kind of simple scheme can be beneficial for legacy cellular networks (e.g., 2G and 3G networks), which have relatively low system capacity compared to WiFi. However, the capacity of latest cellular network (e.g., LTE) is relatively higher and more efficient in terms of the spectral-efficiency (SE). Therefore, off-loading all data traffic to WiFi in the dual coverage area may not always be advantageous; especially, when the user density is much higher under the WiFi coverage.

In order to overcome the shortcoming of mainstream WLAN-first scheme, some Load Balancing schemes have been proposed in literature [15-17]. In these schemes, the traffic is distributed among the available networks, based on different network conditions and objectives. A load balancing scheme, based on fuzzy logic algorithm is proposed in [15] for a 3G and WLAN HetNet, which focuses on the utilisation fairness. In [16], the authors propose a load balancing algorithm to improve network utilization and call blocking probability. A policy based resource management framework is presented in [17] for Cellular-WLAN integrated network, which improves the network utilization by dynamically balancing the offered traffic load via call admission control (CAC) and vertical handoff (VHO). Moreover, the works in [18-20] also propose some CAC policies for a multi-RAT HetNet in order to improve the system performance and balance the load of the network. In [18], the authors propose a CAC scheme for a LTE-WLAN HetNet, where the user's relative preference for the WLAN changes adaptively based on the available resources in 
the WLAN and the relative distribution of cellular users. A CAC algorithm is proposed in [19], which allows the WLAN to limit downward VHOs from the cellular network in order to reduce unnecessary VHO processing. Through numerical and simulation analysis, it has been demonstrated that the proposed CAC scheme reduces the unnecessary VHO processing, while keeping the downward VHO blocking rate within an acceptable limit and maintaining reasonable throughput in the WLAN. In [20], the authors propose a joint-CAC scheme, and Dynamic Bandwidth Adaptation (DBA) scheme. They show that their scheme can maximise the overall system utilization, while keeping the call blocking and dropping rates at acceptable low levels, and satisfying the QoS demands of the diverse services.

In general, the objectives of the existing network selection schemes in literature are limited to optimal resource utilization and/or load balancing. In terms of EE, there are some studies in the literature that focus on mobile devices EE, due to the limited source of energy provided by the battery in such devices. Most notably, in [21], the authors propose a network selection scheme, which optimizes the energy efficiency of mobile devices, with the use of a cost function in a multi-RAT environment that consists of WiFi, WiMax, and 3G networks. An energy-efficient adaptive wireless network interface-selection algorithm is proposed in [22] for mobile devices that are equipped with both $3 \mathrm{G}$ and WiFi network interfaces. While aforementioned studies only focus on the EE of mobile devices, some recent studies consider the EE of the network. As a notable example, the energy-efficient network selection scheme proposed in [9] aims to improve the energy efficiency of the wireless network. In [9], the network selection problem is formulated as a process of an evolutionary game, where the users compete for the data rate from different access networks; and, the network selection by a user is based on the payoff, which is a function of data rate and power consumption. It is assumed in [9] that each network provides a fixed throughput (e.g., $7 \mathrm{Mbps}$ for WLAN and $2 \mathrm{Mbps}$ for $3 \mathrm{G}$ ). However, in reality the network throughput is not fixed and it varies with channel quality. In addition, the power consumption model used in [9], does not capture the realistic total power consumption of base stations (BSs) or APs. For more related work on the network selection in HetNets, please refer to the detailed surveys [7] and [8].

\section{System Model}

This section presents a detail description of the considered system model for the proposed network selection scheme. We consider an operator deployed Cellular-WLAN HetNet, which consists of a single cellular BS and $M$ WiFi APs. Fig. 1 shows an example of the system model. Typically, operators deploy the WiFi APs in high user density areas (e.g., shopping malls and airports, etc), in order to alleviate the traffic burden of the cellular system. Thus, the users are considered to be randomly distributed with higher density in the areas which are within the coverage of each WiFi $\mathrm{AP}$, and lower density in other areas. We focus on downlink data traffic scenario, where each user downloads certain amount of data from the network. It is assumed that service requests follow Poisson Process with average rate of $\lambda$ requests per second.

Typically, each RAT supports a limited number of MCS levels. Hence, we consider that cellular BS supports $K$ levels of MCS, and WiFi AP supports $\hat{K}$ levels. An example is illustrated in Fig. 1 , where each ring depicts a certain MCS level. The system specific overhead is captured with a parameter named "system efficiency" for each type of RAT. It should be noted that the overhead introduced by the underlying multiple access schemes is different for each type of RAT. For example, the overhead caused by Carrier Sense Multiple Access with Collision Avoidance (CSMA/CA) in WiFi is much higher than the signalling overheads in Orthogonal Frequency-Division Multiple 


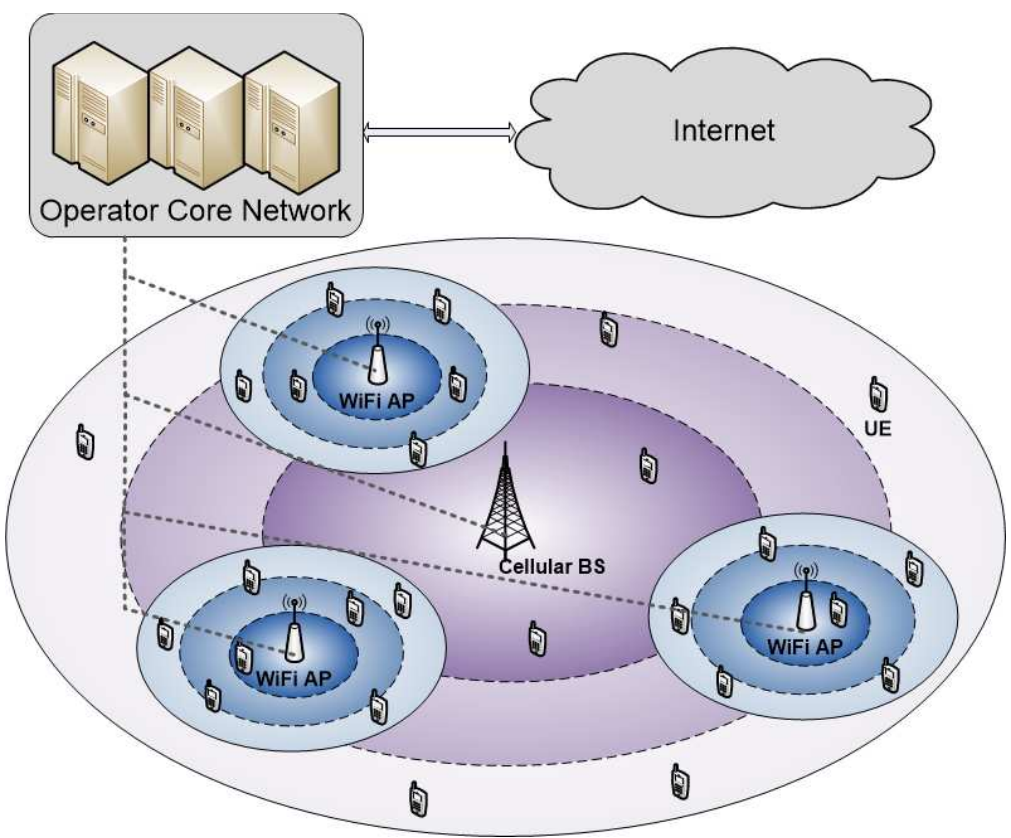

Fig. 1: System model.

Access (OFDMA) in cellular. Typically, the network resource (i.e., system bandwidth) is allocated by a centralised scheduler in cellular networks according to the underlying resource allocation scheme. In contrast to the cellular systems, the radio channel is randomly accessed in WiFi system. This random access method is called Distributed Coordination Function (DCF). A detailed theoretical analysis of DCF mechanism is given in [23]. However, in [23], only single physical layer (PHY) transmission rate is considered. The performance analysis of multi-rate WiFi is carried out in [24] and [25]. In these works, it has been shown that under the DCF mechanism, the throughput of all users attached to the same WiFi AP will be equal and lower than the PHY rate of the lowest rate user. This is referred to as Performance anomaly. The reason for this performance anomaly is that the underlying CSMA/CA mechanism of WiFi, which guarantees equal long term channel access probability for all users [24]. Hence, the WiFi is classified as the throughput fair access network in [26]. Although the users attached to the same AP achieve same throughput, different user combinations (i.e., number of users attached to the AP and their channel conditions) result in distinct throughput value [26]. Hence, the users associated with different WiFi AP may have different throughput.

In order to obtain the network EE, a simplified yet practical linear power model has been suggested in [27]. According to this model, the overall power consumption $P$ of a BS is a linear function of the radiated signal power $P_{t}$. Therefore, it can be derived as $P=P_{0}+\Delta P_{t}$, where $P_{0}$ represents the circuit power consumption, and $\Delta$ is linear coefficient. In addition, when there is no load (i.e., no users served by the BS) the power consumption of BS will be lower than $P_{0}$, which is referred to as idle power consumption. Hence, in this paper, a stated based power consumption model is considered for both cellular BS and WiFi APs (which is given in Section 5.2). With regard to the UE's power consumption, it is assumed that a fixed amount of power is consumed (yet different values for different RATs) by each UE while downloading the data. However, the amount of energy consumed to download certain amount of data by the UE will depend on the time taken to download that data (i.e., it depends on the user's perceived throughput). 


\section{Proposed Network Selection Scheme}

In this section, we propose the ASRAS scheme for an operator deployed Cellular-WLAN HetNet. This scheme aims to improve the network performance in terms of network EE, system throughput, and call blocking probability. In ASRAS, the network selection is stochastically made based on the current network status and channel quality. A probability threshold $\omega(\in[0,1])$ is obtained based on the channel quality (i.e., MCS) of both cellular and WiFi access links, which indicates the preference towards selecting WiFi when the user in dual coverage area. The UE generates a uniform random value $R(\in[0,1])$, and compares $R$ with $\omega$ in order to make the network selection decision. Hence, $\omega$ dictates the selection of WiFi instead of cellular, stochastically. Note that $\omega$ can be interpreted as the expected proportion of users off-loaded to WiFi in the dual coverage area. The network operators can optimise the value of $\omega$ according to the objectives (e.g., system EE, throughput, etc).

The flow chart in Fig. 2 shows the procedure of the proposed scheme. In ASRAS, when a service request is initiated by a UE, the UE first checks whether it is within WiFi coverage or not. If not, the UE checks the load of the cellular BS. If the BS is fully loaded, the request is blocked, otherwise the cellular BS is selected. If the UE is within WiFi coverage, it queries the current network status information (e.g., current load on WiFi AP and cellular BS) from the network. The requested information can be delivered to the UE through standard protocols such as ANDSF or MIH. However, actual implementation of such protocols is out of the scope of this paper. Once the UE receives the network status information, it checks whether the cellular BS and WiFi AP are fully loaded. If both WiFi AP and cellular BS are fully loaded, the request will be blocked. In the case that only either cellular BS or WiFi AP is fully loaded, the UE selects WiFi AP or cellular $\mathrm{BS}$, accordingly. Otherwise, the UE estimates the MCS for both WiFi and cellular, and queries the value of $\omega$ from the network for the estimated MCSs. Then the UE generates a random value $R$ $(\in[0,1])$ and compares it with $\omega$. If $R<\omega$, the UE will select WiFi AP, otherwise, it will choose the cellular BS.

One of the advantages of this scheme is that it eliminates the complex computation on the UE side. At the same time, it allows the operators to influence the network selection decision by adjusting the value for $\omega$ to realize different objectives. However, finding the optimal value of $\omega$ is the most challenging part in the proposed scheme. Thus, we formulate the optimisation problem that yields the optimal $\omega$ as follows:

$$
\begin{aligned}
\text { OPT- } C: \max _{\omega} & C, \\
\text { OPT- } E E: \max _{\omega} & E E, \\
\text { OPT- } P_{b}: \min _{\omega} & p_{b} \\
\text { s.t. } & \omega \in[0,1] .
\end{aligned}
$$

where OPT- $C$, OPT- $E E$ and OPT- $P_{b}$ will result in optimal $\omega$ for maximum network throughput, maximum network EE, and minimum blocking probability, respectively. In the following Section 5, we develop an analytical model based on CTMC to solve these optimisation problems. Using this analytical model, the optimal value of $\omega$ can be computed at a centralised network entity, such as the cellular BS. In addition, since the network selection is stochastically made, the optimal value of $\omega$ does not need to be computed for each arrival of the service request. The value of $\omega$ can be 


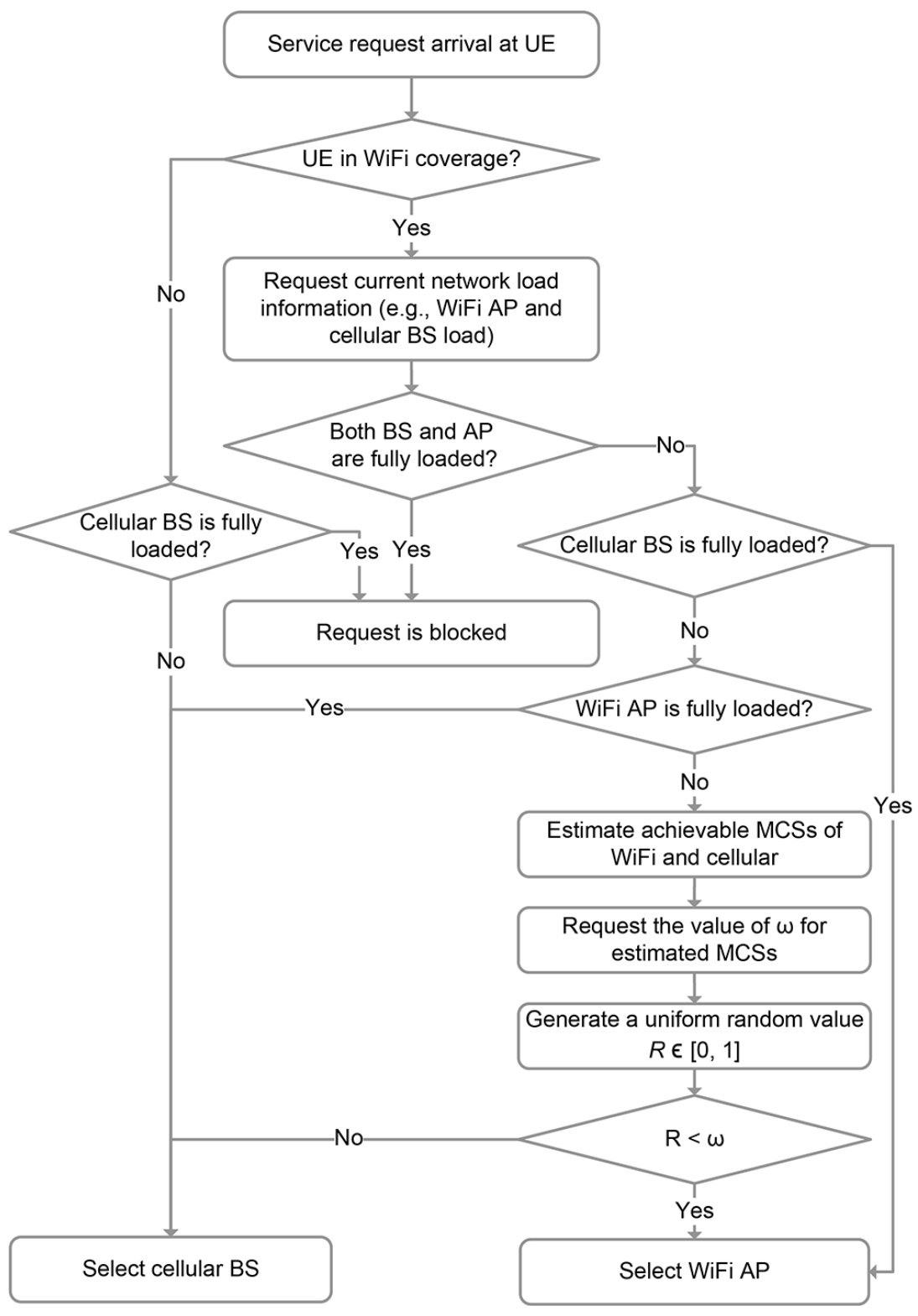

Fig. 2: Proposed ASRAS scheme.

computed offline for various network conditions (e.g., arrival rate, and user density ratio between hot-spot and non-hot-spot area, etc), and stored as a lookup table at the network side (i.e., BS/AP). Hence, the network selection decision can be made without any delay.

\section{Analytical Model}

In this section, we develop an analytical model to solve the optimisation problems in (1). For the following analysis, we denote the notations with superscript $a$ and $b$ that correspond to WiFi and cellular, respectively. The list of major notations used in this analytical model is given in Table 1. Let, $r_{k}^{b}$ denotes the maximum distance from cellular BS that supports the MCS $k(1 \leq k \leq$ $K)$; similarly, the maximum distance from WiFi AP that supports MCS $\hat{k}(1 \leq \hat{k} \leq \hat{K})$ is $r_{\hat{k}}^{a}$. 
Considering that $r_{k}^{b}$ and $r_{\hat{k}}^{a}$ are fixed for a given environment, the system can be modeled with multiple rings [11,18]. Fig. 3 illustrate the ring model, where each ring area corresponds to a MCS that is used to transmit data in that area (i.e., MCS $k$ in cellular and $\hat{k}$ in $\mathrm{WiFi}$ ). $A_{k}^{b}$ represents the ring area where only cellular coverage is available with MCS $k$ and $A_{k, \hat{k}}^{a, m}$ represents the area of intersection with MCS $k$ of cellular and MCS $\hat{k}$ of WiFi AP $m$. For known values of $r_{k}^{b}, r_{\hat{k}}^{a}$ and distance of AP $m$ from BS, the values of $A_{k}^{b}$ and $A_{k, \hat{k}}^{a, m}$ can be easily obtained by geometry for all $k, \hat{k}$ and $m$.

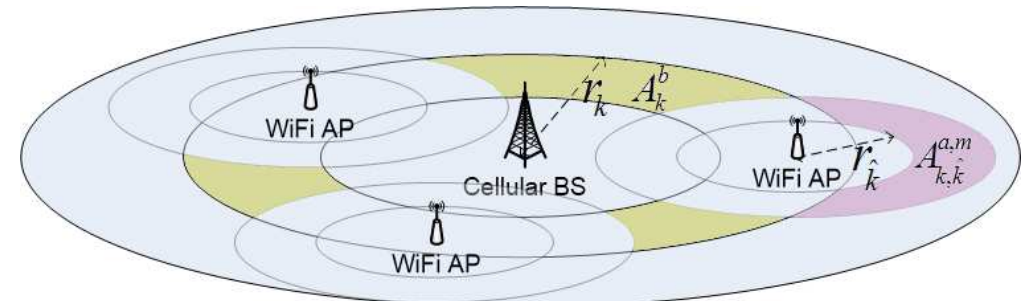

Fig. 3: Ring based model of Cellular-WLAN HetNet.

Since the users are uniformly distributed in both hot-spot and non-hot-spot areas (with higher density in hot-spot areas), the probability of a user being in area $A_{k}^{b}$, and area $A_{k, \hat{k}}^{a, m}$ can be obtained as follows:

$$
\begin{aligned}
p_{k}^{b} & =\frac{A_{k}^{b}}{\sum_{k=1}^{K} A_{k}^{b}+\alpha \sum_{m=1}^{M} \sum_{\hat{k}=1}^{\hat{K}} \sum_{k=1}^{K} A_{k, \hat{k}}^{a, m}}, \\
p_{k, \hat{k}}^{a, m} & =\frac{\alpha A_{k, \hat{k}}^{a, m}}{\sum_{k=1}^{K} A_{k}^{b}+\alpha \sum_{m=1}^{M} \sum_{\hat{k}=1}^{\hat{K}} \sum_{k=1}^{K} A_{k, \hat{k}}^{a, m}},
\end{aligned}
$$

where $\alpha$ is the user density ratio between hot-spot and non-hot-spot areas. Let $n_{k}^{b}$ and $n_{\hat{k}}^{a, m}$ represent the number of users served by the cellular BS and the WiFi AP $m$ with MCS $k$ and MCS $\hat{k}$, respectively. Thus, at any given instance, the system state can be represented as $s:=$ $\left\{n_{1}^{b}, . ., n_{k}^{b}, . . n_{K}^{b}, n_{1}^{a, 1}, . . n_{\hat{k}}^{a, m}, . ., n_{\hat{K}}^{a, M}\right\}$. If the maximum number of simultaneous users that can be served by cellular BS and WiFi AP is $N^{b}$ and $N^{a}$, respectively, the permissible state space $S$ is will be

$$
S=\left\{s \mid \sum_{k=1}^{K} n_{k}^{b} \leq N^{b}, \sum_{\hat{k}=1}^{\hat{K}} n_{\hat{k}}^{a, m} \leq N^{a}\right\} .
$$

Let $b_{u}(s)$ is the portion of the system bandwidth allocated to user $u$ of cellular BS at a given system state $s$, according to the underlying resource allocation scheme (e.g., proportional-fair, round-robin, etc). Hence, the throughput of a user $u$ with MCS $k$ in cellular is

$$
c_{u, k}^{b}(s)=b_{u} B \beta_{k}^{b} \eta^{b}
$$


Table 1 Summary of Notations

\begin{tabular}{|c|c|}
\hline Notation & Description \\
\hline$M$ & Number of WiFi APs \\
\hline$k(\hat{k})$ & MCS index of cellular (WiFi) \\
\hline$K(\hat{K})$ & Number of supported MCS in cellular (WiFi) \\
\hline$r_{k}^{b}\left(r_{\hat{k}}^{a}\right)$ & Max distance from BS (AP) that supports MCS $k(\hat{k})$ \\
\hline$A_{k}^{b}$ & Cellular only area that support MCS $k$ \\
\hline$A_{k, \hat{k}}^{a, m}$ & Intersecting area with MCS $k$ of BS and $\hat{k}$ of AP $m$ \\
\hline$\alpha, \omega$ & User density ratio between hot-spot and non-hot-spot \\
\hline$p_{k}^{b}\left(p_{k, \hat{k}}^{a, m}\right)$ & Probability of a user being in area $A_{k}^{b}\left(A_{k, \hat{k}}^{a, m}\right)$ \\
\hline$n_{k}^{b}\left(n_{\hat{k}}^{a, m}\right)$ & Number of users served by BS (AP $m)$ with MCS $k(\hat{k})$ \\
\hline$N^{b}\left(N^{a}\right)$ & Maximum number of users can be served by BS (AP) \\
\hline$\beta_{k}^{b}$ & Spectral efficiency of cellular at MCS $k$ \\
\hline$\beta_{\hat{k}}^{a}$ & PHY rate of WiFi at MCS $\hat{k}$ \\
\hline$\eta^{b}\left(\eta^{a}\right)$ & System efficiency of cellular (WiFi) \\
\hline$B$ & Cellular system bandwidth \\
\hline$b_{u}$ & Portion of allocated system bandwidth to user $u$ by BS \\
\hline$c^{b}(s)$ & Throughput of cellular BS at state $s$ \\
\hline$c^{a, m}(s)$ & Throughput of WiFi AP $m$ at state $s$ \\
\hline$c_{u, k}^{b}(s)$ & Average throughput of user served by BS with MCS $k$ \\
\hline$c_{u}^{a, m}(s)$ & Average throughput of user served by WiFi AP $m$ \\
\hline$\lambda$ & Service request arrival rate in the system \\
\hline$\lambda_{k}^{b}\left(\lambda_{k, \hat{k}}^{a, m}\right)$ & Service request arrival rate in area $A_{k}^{b}\left(A_{k, \hat{k}}^{a, m}\right)$ \\
\hline$\varepsilon \varepsilon^{n, n}$ & Average amount of data download per user \\
\hline$\mu_{k}^{b}\left(\mu^{a, m}\right)$ & Service completion rate at BS with MCS $k(\mathrm{WiFi}$ AP $m)$ \\
\hline$\omega_{k \hat{k}}$ & Probability of selecting WiFi in area $A_{k \hat{k}}^{a, m}$ \\
\hline$\psi_{k}^{b}$ & Rate of the state transition of $\left(n_{k}^{b} \rightarrow n_{k}^{k}+1\right)$ \\
\hline$\phi_{k}^{b}$ & Rate of the state transition of $\left(n_{k}^{b} \rightarrow n_{k}^{b}-1\right)$ \\
\hline$\psi_{\hat{k}}^{a, m}$ & Rate of the state transition of $\left(n_{\hat{k}}^{a, m} \rightarrow n_{\hat{k}}^{a, m}+1\right)$ \\
\hline$\phi_{\hat{k}}^{\alpha, m}$ & Rate of the state transition of $\left(n_{\hat{k}}^{a}, m \rightarrow n_{\hat{k}}^{a, m}-1\right)$ \\
\hline$\pi(s)$ & Steady state probability of state $s$ \\
\hline$p_{b}$ & System call blocking probability \\
\hline$p_{b}^{b}\left(p_{h}^{a, m}\right)$ & Call blocking probability of cellular BS (WiFi AP $m$ ) \\
\hline$P_{I}^{b}\left(P_{I}^{a}\right)$ & Idle power consumption of BS (AP) \\
\hline$P_{0}^{b}\left(P_{0}^{a}\right)$ & Static power consumption of BS (AP) \\
\hline$P_{t}^{b}\left(P_{t}^{a}\right)$ & Transmit power of BS (AP) \\
\hline$\Delta^{b}\left(\Delta^{a}\right)$ & Linear coefficient of power consumption of BS (AP) \\
\hline$P_{u}^{b}\left(P_{u}^{a}\right)$ & UE power consumption when served by BS (AP) \\
\hline
\end{tabular}

where $B$ is the total system bandwidth of cellular BS, $\beta_{k}^{b}$ is the spectral efficiency (SE) of BS at MCS $k$, and $\eta^{b}$ is the system efficiency that reflects all system specific overheads of cellular system (e.g., signalling, protocol headers, etc). Hence, the system throughput of cellular BS (i.e., sum-rate 
of UEs), for a given system state $s$ is

$$
c^{b}(s)=\sum_{k=1}^{K} n_{k}^{b} c_{u, k}^{b}(s) .
$$

Since the channel is randomly accessed in WiFi, the throughput model of a user associated with a WiFi AP will be deferent to that of a user associated to cellular. It has been shown that the throughput of all users attached to the same WiFi AP will be equal and lower than the lowest physical layer transmission rate of all users $[24,25]$. This is due to the fact that the CSMA/CA mechanism of WiFi guarantees equal long term channel access probability for all users [24]. However, throughput of an AP depends on the number of users served with each physical layer transmission rate. Hence, the throughput of the WiFi AP $m$ at system state $s$ can be modeled as [26]

$$
c^{a, m}(s)=\frac{\sum_{\hat{k}=1}^{\hat{K}} n_{\hat{k}}^{a, m} \beta_{\hat{k}}^{a}}{\sum_{\hat{k}=1}^{K} n_{\hat{k}}^{a, m}} \eta^{a},
$$

where $\beta_{\hat{k}}^{a}$ is the physical layer transmission rate of WiFi with MCS $\hat{k} . \eta^{a}$ is the system efficiency of WiFi that captures all the system specific overheads of WiFi including the overhead caused by the random access. Thus, the average user throughput of user $u$ served by WiFi AP $m$, at system state $s$ will be

$$
c_{u}^{a, m}(s)=\frac{c^{a, m}(s)}{\sum_{\hat{k}=1}^{\hat{K}} n_{\hat{k}}^{a}} .
$$

Table 2 Events and State Transitions of CTMC

\begin{tabular}{|c|l|c|c|l|}
\hline Event & Description & State Transition & Rate & Condition \\
\hline \hline (a) & An arrival in area $A_{k}^{b}$ & $n_{k}^{b} \rightarrow n_{k}^{b}+1$ & $\lambda_{k}^{b}$ & $\sum_{k=1}^{K} n_{k}^{b}<N^{b}$ \\
\hline (b) & $\begin{array}{l}\text { An arrival in area } A_{k, \hat{k}}^{a, m} \\
\text { and select WiFi }\end{array}$ & $n_{\hat{k}}^{a, m} \rightarrow n_{\hat{k}}^{a, m}+1$ & $\lambda_{k, \hat{k}}^{a, m} \omega_{k, \hat{k}}$ & $\sum_{\hat{k}=1}^{\hat{K}} n_{\hat{k}}^{a, m}<N^{a}$ \\
\hline (c) & $\begin{array}{l}\text { An arrival in area } A_{k, \hat{k}}^{a, m} \\
\text { and select cellular }\end{array}$ & $n_{k}^{b} \rightarrow n_{k}^{b}+1$ & $\lambda_{k, \hat{k}}^{a, m}\left(1-\omega_{k, \hat{k}}\right)$ & $\sum_{k=1}^{K} n_{k}^{b}<N^{b}$ \\
\hline (d) & $\begin{array}{l}\text { A user served by BS with MCS } k \\
\text { completes the session }\end{array}$ & $n_{k}^{b} \rightarrow n_{k}^{b}-1$ & $n_{k}^{b} \mu_{k}^{b}$ & None \\
\hline (e) & $\begin{array}{l}\text { A user served by WiFi AP } m \text { with } \\
\text { MCS } \hat{k} \text { completes the session }\end{array}$ & $n_{\hat{k}}^{a, m} \rightarrow n_{\hat{k}}^{a, m}-1$ & $n_{\hat{k}}^{a, m} \mu^{a, m}$ & None \\
\hline
\end{tabular}

Since the service requests from the users follow a Poisson Process with an average rate of $\lambda$, the arrival rate in area $A_{k}^{b}$ and $A_{k, \hat{k}}^{a, m}$ will be $\lambda_{k}^{b}=\lambda p_{k}^{b}$ and $\lambda_{k, \hat{k}}^{a, m}=\lambda p_{k, \hat{k}}^{a, m}$ respectively. The service time (i.e., dwell-time) $1 / \mu_{k}^{b}\left(1 / \mu^{a, m}\right)$ of a user with MCS $k$ of cellular (WiFi) will be,

$$
\frac{1}{\mu_{k}^{b}}=\frac{\varepsilon}{c_{u, k}^{b}}
$$




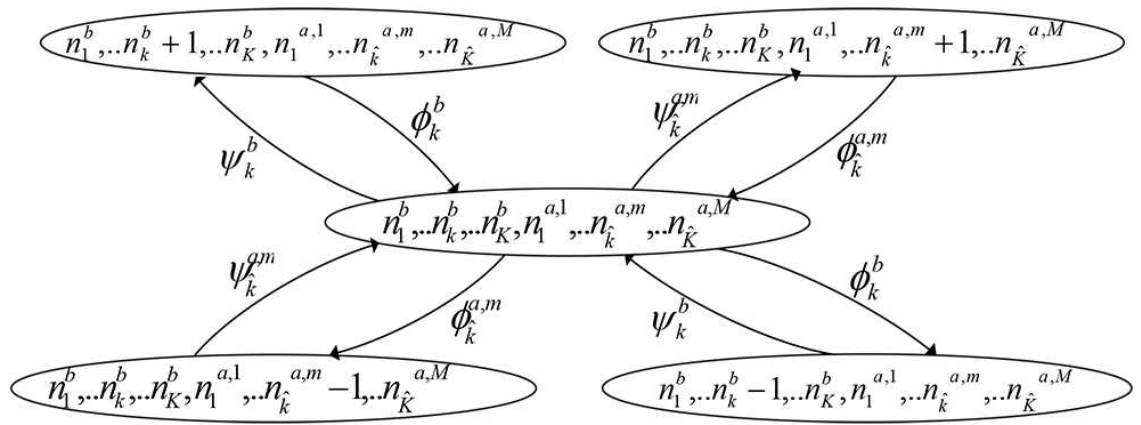

Fig. 4: State transition diagram of CTMC.

$$
\frac{1}{\mu^{a, m}}=\frac{\varepsilon}{c_{u}^{a, m}}
$$

where $\varepsilon$ is the average amount of data downloaded by each user. Hence, the system can be modeled as a CTMC. Fig. 4 shows the state transition diagram of the CTMC. The events and the relevant state transitions due to service request arrival and service completion are given in Table 2, where $\omega_{k, \hat{k}}\left(0 \leq \omega_{k, k} \leq 1\right)$ is the probability of selecting WiFi in area $A_{k, \hat{k}}^{a, m}$. The events (a) and (c) in Table 2 will result in the state transition of $\left\{. ., n_{k}^{b}, \ldots n_{\hat{k}}^{a, m}, ..\right\} \rightarrow\left\{. ., n_{k}^{b}+1, \ldots n_{\hat{k}}^{a, m}, ..\right\}$; hence, the rate of this state transition in CTMC will be

$$
\psi_{k}^{b}=\lambda_{k}^{b}+\lambda_{k, \hat{k}}^{a, m}\left(1-\omega_{k, \hat{k}}\right) .
$$

Event (b) results in $\left\{. ., n_{k}^{b}, \ldots n_{\hat{k}}^{a, m}, ..\right\} \rightarrow\left\{. ., n_{k}^{b}, \ldots n_{\hat{k}}^{a, m}+1, ..\right\}$; hence, the transition rate is

$$
\psi_{\hat{k}}^{a, m}=\lambda_{k, \hat{k}}^{a, m} \omega_{k, \hat{k}}
$$

Similarly, transition $\left\{. ., n_{k}^{b}, \ldots n_{\hat{k}}^{a, m}, ..\right\} \rightarrow\left\{. ., n_{k}^{b}-1, \ldots n_{\hat{k}}^{a, m}, ..\right\}$ will be a result of event (d), whereas the transition $\left\{. ., n_{k}^{b}, \ldots n_{\hat{k}}^{a, m}, ..\right\} \rightarrow\left\{. ., n_{k}^{b}, \ldots n_{\hat{k}}^{a, m}-1, ..\right\}$ will be a result of event (e); thus, the state transition rates are

$$
\begin{gathered}
\phi_{k}^{b}=n_{k}^{b} \mu_{k}^{b} . \\
\phi_{\hat{k}}^{a, m}=n_{\hat{k}}^{a, m} \mu^{a, m},
\end{gathered}
$$

respectively. Let $\pi(s)$ be the steady state probability of state $s$. The steady state probabilities are related to each other by the global balance equations that are obtained form the state transition diagram. By combining the global balance equations with the normalisation condition $\sum_{s \in S} \pi(s)=1$, the steady state probabilities $\pi$ can be calculated. We use the successive over relaxation (SOR) algorithm to calculate the $\pi$. The pseudo code of the SOR algorithm is given in Appendix 9.1. From $\pi$, the system performances can be obtained as described in the following subsections.

\subsection{System throughput}

The system performance in terms of total system throughput $C$ (i.e., the sum-rate of the BS and the APs) at steady state can be obtained from (6) and (7) as: 


$$
C=\sum_{s \in S} \pi(s)\left(\sum_{m=1}^{M} c^{a, m}(s)+c^{b}(s)\right) .
$$

\subsection{Network energy efficiency}

In order to evaluate the $\mathrm{EE}$, first, we define the following state spaces $X_{0}^{b}$ and $X_{0}^{a, m}$, where the number of users served by the cellular BS and the WiFi AP $m$ is zero, respectively. Thus,

$$
\begin{aligned}
X_{0}^{b} & =\left\{s \in S \mid n_{k}^{b}=0, \forall k\right\}, \\
X_{0}^{a, m} & =\left\{s \in S \mid n_{\hat{k}}^{a, m}=0, \forall \hat{k}\right\} .
\end{aligned}
$$

Then, the state based power consumption of the cellular BS $P^{b}(s)$ and the WiFi AP $m P^{a, m}(s)$ at state $s$ can be represented as follows:

$$
\begin{gathered}
P^{b}(s)= \begin{cases}P_{I}^{b} & \forall s \in X_{0}^{b}, \\
P_{0}^{b}+\Delta^{b} P_{t}^{b} & \text { otherwise, }\end{cases} \\
P^{a, m}(s)= \begin{cases}P_{I}^{a} & \forall s \in X_{0}^{a, m}, \\
P_{0}^{a}+\Delta^{a} P_{t}^{a} & \text { otherwise, }\end{cases}
\end{gathered}
$$

where $P_{I}^{b}\left(P_{I}^{a}\right), P_{0}^{b}\left(P_{0}^{a}\right), P_{t}^{b}\left(P_{t}^{a}\right)$ and $\Delta^{b}\left(\Delta^{a}\right)$ represent the idle power consumption, circuit power consumption, transmit power, and linear coefficient of cellular BS (WiFi AP), respectively. From (15), (18) and (19), the total network EE at steady state can be obtained as,

$$
E E=\frac{C}{\sum_{s \in S} \pi(s)\left(\sum_{m=1}^{M} P^{a, m}(s)+P^{b}(s)\right)} .
$$

\subsection{Call blocking probability}

The state spaces $X_{N}^{b}$ and $X_{N}^{a, m}$ are defined as the number of users served by the cellular BS and the WiFi AP $m$ is $N^{b}$ and $N^{a}$, respectively. Thus,

$$
\begin{gathered}
X_{N}^{b}=\left\{s \in S \mid \sum_{k=1}^{K} n_{k}^{b}=N^{b}\right\}, \\
X_{N}^{a, m}=\left\{s \in S \mid \sum_{\hat{k}=1}^{\hat{K}} n_{\hat{k}}^{a, m}=N^{a}\right\} .
\end{gathered}
$$

When the system in state $s \in X_{N}^{b}\left(s \in X_{N}^{a, m}\right)$, any arrival at cellular BS (WiFi AP $m$ ) will be blocked. Hence, the call blocking probabilities of a user at cellular BS and WiFi AP $m$ can be obtained as

$$
p_{b}^{b}=\sum_{s \in X_{N}^{b}} \pi(s)\left(\sum_{k=1}^{K}\left[p_{k}^{b}+\sum_{\hat{k}=1}^{\hat{K}} p_{k, \hat{k}}^{a, m}\left(1-\omega_{k, \hat{k}}(s)\right)\right]\right)
$$




$$
p_{b}^{a, m}=\sum_{s \in X_{N}^{a, m}} \pi(s)\left(\sum_{k=1}^{K} \sum_{\hat{k}=1}^{\hat{K}} p_{k, \hat{k}}^{a, m} \omega_{k, \hat{k}}(s)\right),
$$

respectively. Since these probabilities are mutually exclusive (i.e., a user can only be blocked by either cellular or WiFi, depending on the selected network), the system call blocking probability $p_{b}$ will be

$$
p_{b}=\sum_{m=1}^{M} p_{b}^{a, m}+p_{b}^{b}
$$

\subsection{User equipment energy efficiency}

Since the analysis is for downlink scenario, the power consumption of a UE considered to be fixed while downloading the data. However, it depends on the associated network. Hence the UE power consumption $P_{u}$ is given as

$$
P_{u}= \begin{cases}P_{u}^{a} & \text { if the UE is accociated with WiFi, } \\ P_{u}^{b} & \text { if the UE is accociated with cellular. }\end{cases}
$$

Thus, the average UE energy consumption at steady state, to download the data from WiFi AP $m$ and cellular BS can be obtained as

$$
\begin{gathered}
E C_{u}^{a, m}=P_{u}^{a} \sum_{s \in S} \pi(s) \mu^{a, m}(s)\left(\sum_{k=1}^{K} \sum_{\hat{k}=1}^{\hat{K}} p_{k \hat{k}}^{a, m} \omega_{k, \hat{k}}(s)\right), \\
E C_{u}^{b}=P_{u}^{b} \sum_{s \in S} \pi(s)\left(\sum_{k=1}^{K} \mu_{k}^{b}(s)\left[p_{k}^{b}+\sum_{\hat{k}=1}^{\hat{K}} p_{k, \hat{k}}^{a, m}\left(1-\omega_{k, \hat{k}}(s)\right)\right]\right),
\end{gathered}
$$

respectively. From (27) and (28), the average EE of UE when it is served by WiFi and cellular will be

$$
\begin{aligned}
& E E_{u}^{a}=\frac{\varepsilon}{E C_{u}^{a}}, \\
& E E_{u}^{b}=\frac{\varepsilon}{E C_{u}^{b}},
\end{aligned}
$$

respectively.

By utilising the equations (15), (20), and (25) in this analytical model, the optimization problems OPT- $C$, OPT- $E E$ and OPT- $P_{b}$ in (1) can be solved via exhaustive search method, respectively. The details of the search algorithm is given in Appendix 9.2. In addition, the average UE EE can be analytically obtained from (29) or (30), depending on whether the UE is served by WiFi/cellular. The complexity of the search algorithm is given by $\mathcal{O}\left((1 / \varsigma)^{K \hat{K}}\right)$, where $\varsigma$ is the step size of $\omega$ per iteration. Although the complexity of this algorithm exponentially increases with $K$ and $\hat{K}$, as mentioned earlier the optimal value of $\omega$ does not need to be computed for each arrival of the 
service request since the network selection is stochastically made. The value of $\omega$ can be computed offline for various network conditions (e.g., arrival rate, and user density ratio between hot-spot and non-hot-spot area), and stored as a lookup table at the BS and AP. Thus, there won't be any delay in the network selection decision due to computational complexity.

\section{Performance Evaluation}

The performance of the proposed network selection scheme is evaluated in this section, and compared with two benchmark schemes, namely, WLAN-first and Load Balancing. The system performances are evaluated analytically (by the analytical model proposed in Section 5), and in a system level simulator that is developed in MATLAB. The considered network architecture for the simulation study is depicted in Fig. 1, and the system parameter settings are given in Table 3. In the simulations, the UEs are uniformly distributed in the coverage area, with different densities between hot-spot and non-hot-spot areas according to the parameter $\alpha$. The time interval between consecutive service requests from the UEs follows exponential distribution with parameter $\lambda$ (i.e., follows Poisson Process). Two scenarios are considered for the performance evaluation. In the first scenario, the user density ratio between hot-spot and non-hot-spot area is fixed (i.e., $\alpha=10$ ), but the traffic arrival rate varies. In the second scenario, the arrival rate is fixed (i.e., $\lambda=30$ ), but the user density ratio varies. In both scenarios, three WiFi APs are deployed per cellular BS (i.e., $M=3$ ), which are uniformly deployed with a fixed distance of $150 \mathrm{~m}$ from the BS. Note that for the analytical evaluation of the benchmark schemes, the preference values are obtained as follows:

$$
\omega_{k, \hat{k}}= \begin{cases}1 & \text { for WLAN-first } \forall k, \hat{k}, \\ \frac{N^{a} M}{N^{a} M+N^{b}} & \text { for Load Balancing } \forall k, \hat{k} .\end{cases}
$$

This is due to the fact that in the mainstream WLAN-first scheme, the UE always tries to connect to WiFi for data service whenever the WiFi coverage is available; hence, the probability of selecting WiFi is always 1. For the Load Balancing scheme, in general, the load will be distributed based on the network capacity (i.e., the number of users/flows that can be served by each network). In addition, for the sake of fairness, it is assumed that the resources are equally allocated amongst the associated users in the cellular system (i.e., the system bandwidth is equally shared in cellular system) in all considered schemes.

\subsection{System throughput}

Fig. 5 shows the system performance in terms of system throughput against varied arrival rate $(\lambda)$ and the user density ratio $(\alpha)$. In this figure, the WLAN-first scheme is denoted as "WF" and the Load Balancing scheme is denoted as "LB". In addition, analytically obtained results are denoted as "XX(An)" and the results obtained through simulations are denoted as "XX(Sim)", where "XX" represents the corresponding network selection scheme. From this figure, it can be seen that the analytical results match the results from simulations very well. Fig. 5(a) clearly demonstrates the inefficiency of mainstream WLAN-first and the Load Balancing schemes, against increased arrival rate. This is due to the fact that in WLAN-first scheme, WiFi APs will be highly congested due to high user density in the hot-spot areas while the cellular BS is not fully utilised. In Load Balancing scheme, since the system load is evenly distributed, the performance is higher than WLAN-first. However, variation in channel quality is not considered in Load Balancing. Hence, the proposed scheme performs best. In Fig. 5(b), we can find that the performance of WLAN-first decreases 
Table 3 System Parameter Settings

\begin{tabular}{|c|c||c|c|}
\hline Parameter & Value & Parameter & Value \\
\hline \hline$K$ & 3 & $r_{k}$ & $\{100,200,300\}(\mathrm{m})$ \\
\hline$\hat{K}$ & 2 & $r_{\hat{k}}$ & $\{50,100\}(\mathrm{m})$ \\
\hline$N^{b}$ & 10 & $\beta_{k}$ & $\{5.1,2.4,0.6\}(\mathrm{bits} / \mathrm{Hz} / \mathrm{s})$ \\
\hline$N^{a}$ & 5 & $\beta_{\hat{k}}^{a}$ & $\{24,6\}(\mathrm{Mbps})$ \\
\hline$\eta^{a}$ & 0.4 & $\lambda$ & $5-50($ Variable $)$ \\
\hline$\eta^{b}$ & 0.75 & $\alpha$ & $2-20($ Variable) \\
\hline$B$ & $20 \mathrm{Mhz}$ & $\varepsilon$ & $1 \mathrm{Mbits}$ \\
\hline$P_{I}^{b}$ & $100 \mathrm{~W}$ & $P_{I}^{a}$ & $10 \mathrm{~W}$ \\
\hline$P_{0}^{b}$ & $130 \mathrm{~W}$ & $P_{0}^{a}$ & $10 \mathrm{~W}$ \\
\hline$P_{t}^{b}$ & $20 \mathrm{~W}$ & $P_{t}^{a}$ & $2 \mathrm{~W}$ \\
\hline$\Delta^{b}$ & 4.7 & $\Delta^{a}$ & 2.5 \\
\hline
\end{tabular}

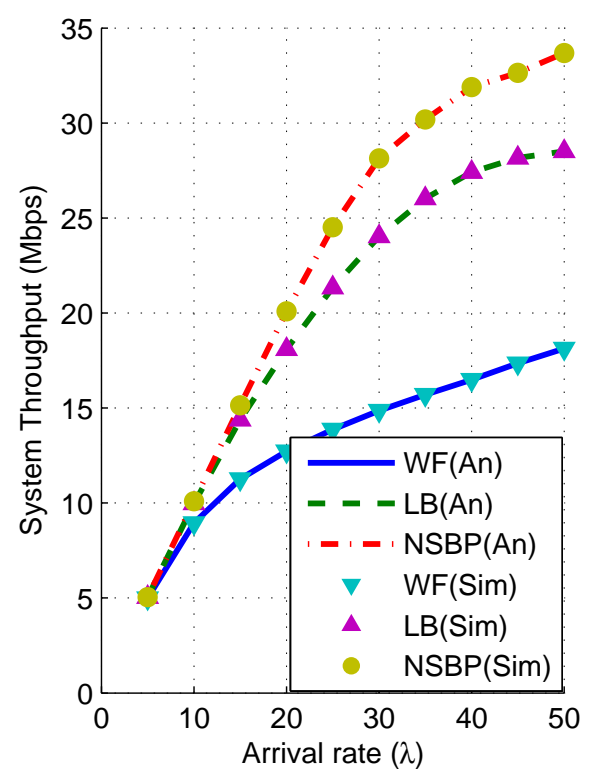

(a)

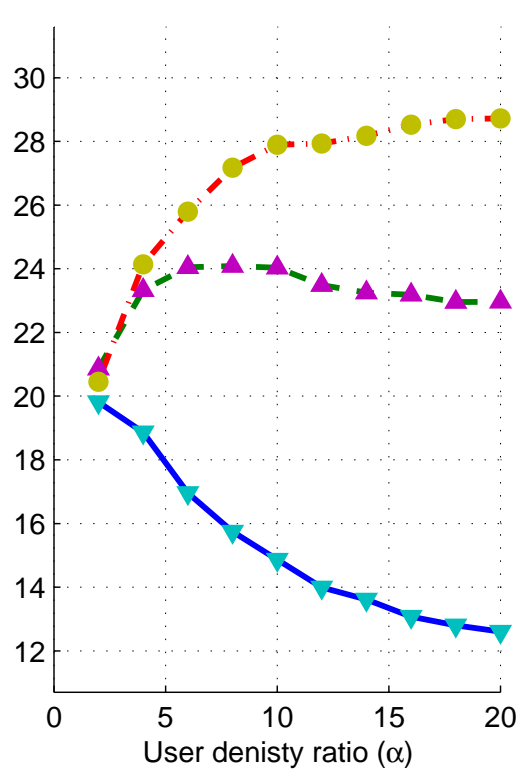

(b)

Fig. 5: System throughput vs. Arrival rate $(\lambda)$ and user density ratio $(\alpha)$.

as $\alpha$ increases. This is due to the fact that when the user density in hot-spot area increases, WiFi becomes heavily congested and cellular becomes under utilised, since all users in dual coverage select WiFi. As a consequence, the system performance deteriorates. In the Load Balancing scheme, the performance increases with increased $\alpha$ up to a certain value of $\alpha$, and then it begins to deteriorate. On the other hand, the performance of the proposed scheme, continuously increases within the whole range of investigated $\alpha$, while demonstrating superior performance compared to both benchmarks. This is due to the fact that in the proposed scheme, the value of $\omega$ is optimized according to the status of the network, and the channel quality. 


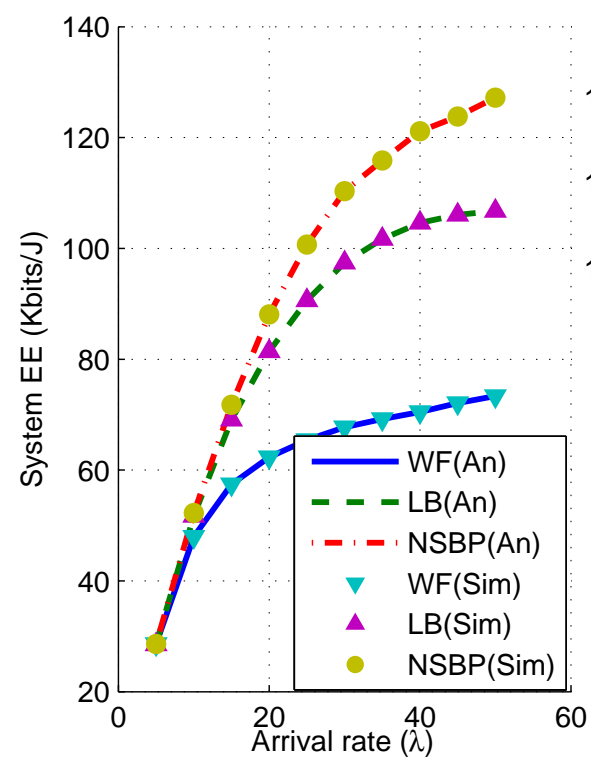

(a)

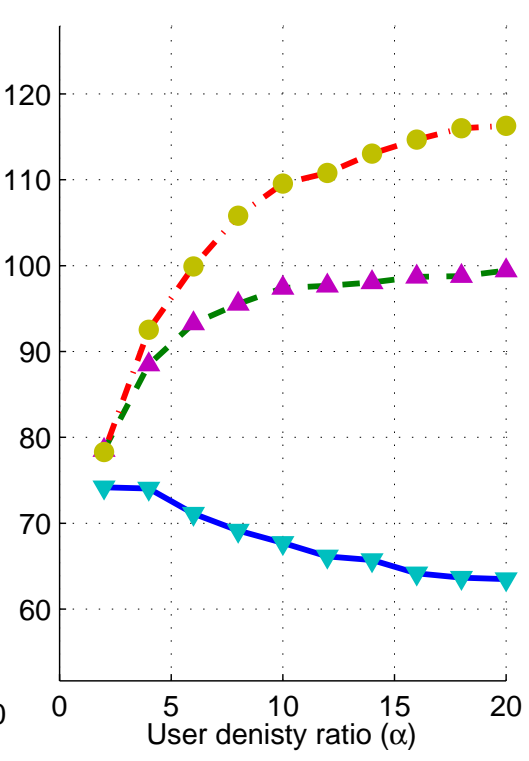

(b)

Fig. 6: System EE vs. Arrival rate $(\lambda)$ and user density ratio $(\alpha)$.

\subsection{Network EE}

Fig. 6 shows the system performance in terms of network EE against increasing $\lambda$ and $\alpha$. In this figure, we can notice similar trend in performance compared to the throughput. This is due to the fact that the system power consumption does not significantly vary when both the AP and the BS are active. In addition, the period when neither AP nor BS is active (i.e., not serving any user) is relatively low. However, in Fig 6(b), we can observe that the system EE of Load Balancing scheme is continuously increasing for whole range of $\alpha$, in contrast to the system throughput. Since the value of $\omega$ is optimized in the proposed scheme, it outperforms the other two benchmarks in both scenarios.

\subsection{Call Blocking probability}

Fig. 7 shows the performance in terms of call blocking probability of the system against increasing $\lambda$ and $\alpha$. From Fig. 7(a), we can observe that the call blocking probability increases when the arrival rate increases for all three schemes. However, the blocking probability in WLAN-first is much higher than the other two schemes. This is due to the fact that the WiFi is selected regardless of the status of the WiFi AP in WLAN-first. Thus, the possibility of AP become fully loaded is high. As a result, the blocking probability in WiFi will be high. The proposed scheme achieves the lowest blocking probability among the three schemes, due to the fact that the state of the WiFi is considered together with channel quality variations. In Fig. 7(b), we see the inverse effect to the system throughput for the blocking probability. The blocking probability increases as $\alpha$ increases in WLAN-first. In the Load Balancing scheme, it decreases first until $\alpha$ increases to certain value and then it increases. The blocking probability is lowest and continuously decreases with the proposed scheme. 


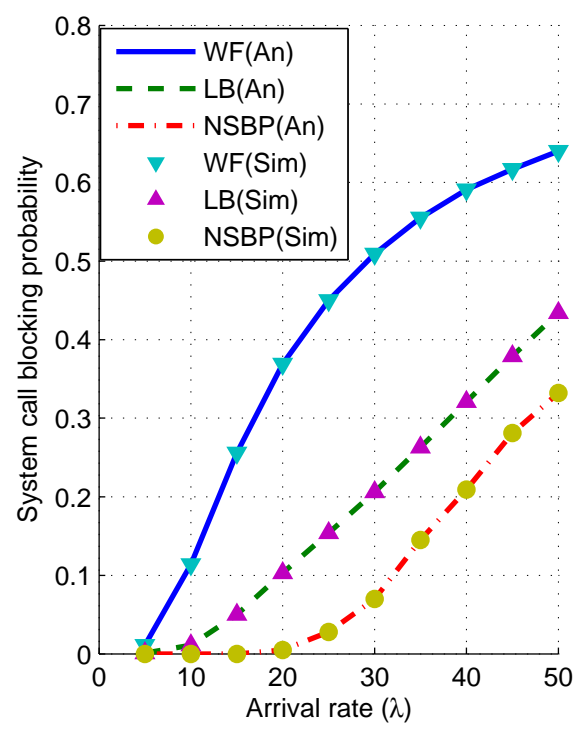

(a)

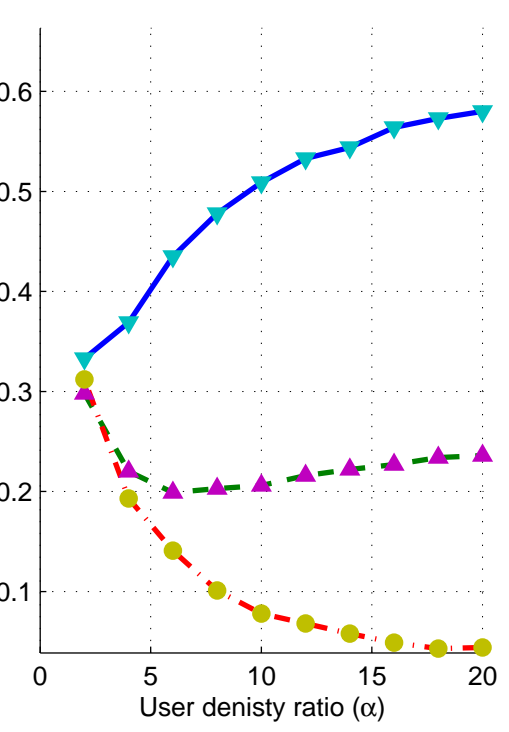

(b)

Fig. 7: Call blocking probability vs. Arrival rate $(\lambda)$ and user density ratio $(\alpha)$.

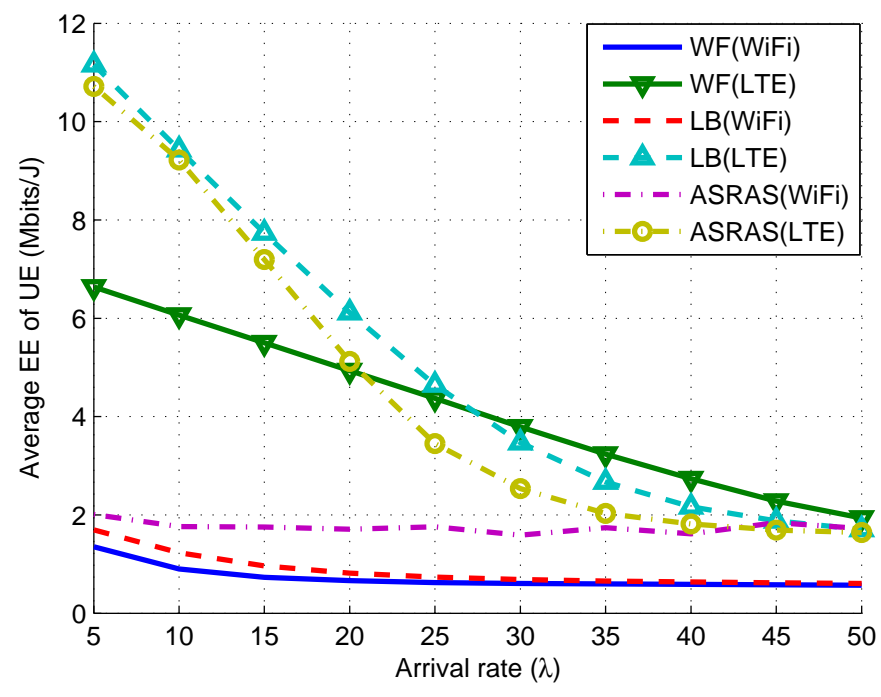

Fig. 8: Average UE EE vs. Arrival rate $(\lambda)$.

\subsection{User equipment $E E$}

In this subsection, we evaluate the EE of UEs against increasing arrival rate when the objective of the proposed scheme is system EE, and compare with the performance of the benchmark schemes. Fig. 8 shows the average UE's EE that is served by the WiFi AP and the cellular BS against increasing arrival rate. As we can observe from this figure, in all three schemes, average UE's EE that is served by WiFi is lower than that of served by cellular. The main reason behind this trend is that the throughput of WiFi AP is lower than cellular BS, at the same time the user density in WiFi (i.e., hot-spot) is much higher; hence, average user throughput of WiFi user is lower than cellular user. Thus, average dwell-time of a UE served by WiFi AP is higher than that of cellular 
BS. Therefore, users spend lower energy to download same amount of data in cellular than WiFi. In addition, we can observe that in the WLAN-first scheme, the average EE of WiFi user is worst among the three schemes in comparison, while the proposed scheme performs best. However, EE of a cellular user is almost similar in the proposed and the Load Balancing schemes; whereas in WLAN-first, it is lower at low arrival rate and better at high arrival rate compared to both proposed and Load Balancing schemes. In general, the UE's EE is better in the proposed scheme compared to the benchmark schemes, although the objective of the proposed scheme is network EE.

\section{Conclusion}

In this paper, we proposed a novel Adaptive Stochastic Radio Access Selection (ASRAS) scheme for Cellular-WLAN HetNet. This scheme implements a stochastic network selection policy that assigns certain probability for selecting WLAN according to the status of the network in terms of load and signal quality. This approach helps to improve the system performance in terms of network throughput, energy efficiency (EE) or call blocking probability. One of the main advantages in this scheme is that it eliminates the complex computation on the UE side. At the same time, it allows the operators to influence the network selection decision by setting the appropriate probability of selecting WLAN according to different objectives. An analytical model is proposed for the Cellular-WLAN HetNet, that considers system specific overhead and limitations as well as state based total power consumption models for each network, in order to compute the optimal probability value for selecting WLAN network. The proposed analytical model is thoroughly validated against simulation results. In addition, comprehensive performance analysis is carried out to compare the performance of the proposed scheme with that of two mainstream network selection schemes, namely, WLAN-first and Load Balancing. Both the analytical and simulation results demonstrate the superiority of the proposed scheme over the compared mainstream schemes.

The main scope of this paper is an operator deployed Cellular-WLAN HetNet that consists of single cellular BS and multiple WiFi APs. As a future direction, this work can be extend to include multi-cell scenario. However, when considering multi-cell scenario, the interference caused by neighbouring cells has to be taken in to account.

\section{References}

[1] S. Landström, A. Furuskãr, K. Johansson, L. Falconetti, and F. Kronestedt, "Heterogeneous networks-increasing cellular capacity," Ericsson Review, p. 4, Feb 2011.

[2] D. Feng, C. Jiang, G. Lim, J. Cimini, L.J., G. Feng, and G. Li, "A survey of energy-efficient wireless communications," Communications Surveys Tutorials, IEEE, vol. 15, no. 1, pp. 167178, Jan 2013.

[3] "Integration of cellular and Wi-Fi networks," 4G Americas, www.4gamericas.org, Tech. Rep., Sep 2013.

[4] S. Rudd and P. Kendall, "Carrier Wi-Fi roaming: Obstacles are delaying next generation hotspots (NGH) but operators can begin service today," Strategy Analytics, Tech. Rep., Apr 2014.

[5] P. Kosmides, A. Rouskas, and M. Anagnostou, "Network selection in heterogeneous wireless environments," in Proc. 18th Int Telecommunications (ICT) Conf, 2011, pp. 250-255. 
[6] R. Chai, W.-G. Zhou, Q.-B. Chen, and L. Tang, "A survey on vertical handoff decision for heterogeneous wireless networks," in Proc. IEEE Youth Conf. Information, Computing and Telecommunication YC-ICT '09, 2009, pp. 279-282.

[7] L. Wang and G.-S. Kuo, "Mathematical modeling for network selection in heterogeneous wireless networks a tutorial," Communications Surveys Tutorials, IEEE, no. 1, pp. 271-292, Jan 2013.

[8] A. Sabbagh, R. Braun, and M. Abolhasan, "A comprehensive survey on rat selection algorithms for heterogeneous networks," World Academy of Science, vol. 49, pp. 141-145, 2011.

[9] C. Qian, C. Luo, F. Hao, L. Yang, and G. Min, "Energy-efficient dynamic network selection in heterogeneous wireless networks," in Green Computing and Communications (GreenCom), 2013 IEEE and Internet of Things (iThings/CPSCom), IEEE International Conference on and IEEE Cyber, Physical and Social Computing, Aug 2013, pp. 71-76.

[10] J. Andrews, S. Singh, Q. Ye, X. Lin, and H. Dhillon, "An overview of load balancing in HetNets: old myths and open problems," Wireless Communications, IEEE, vol. 21, no. 2, pp. 18-25, April 2014.

[11] S. H. Kim, H. B. Jung, and D. K. Kim, "A ring-based performance analysis for a call admission control in an lte/wifi heterogeneous network," in Communications (APCC), 2011 17th Asia-Pacific Conference on, Oct 2011, pp. 84-89.

[12] Access Network Discovery and Selection Function (ANDSF) and Management Object (MO), 3GPP Std. 3GPP TS 24.312, 2013.

[13] IEEE Standard for Local and Metropolitan Area Networks- Part 21: Media Independent Handover, IEEE Std. 802.21-2008, 2009.

[14] W. Song, H. Jiang, and W. Zhuang, "Performance analysis of the WLAN-First scheme in Cellular/WLAN interworking," Wireless Communications, IEEE Transactions on, vol. 6, no. 5, pp. 1932-1952, May 2007.

[15] S. Jin, W. Xuanli, and S. Xuejun, "Load balancing algorithm with multi-service in heterogeneous wireless networks," in Communications and Networking in China (CHINACOM), 2011 6th International ICST Conference on, Aug 2011, pp. 703-707.

[16] J. Ha, J. Kim, J. up Kim, and S.-H. Kim, "Dynamic load balancing architecture in heterogeneous wireless network environment," in Communications and Information Technology, 2009. ISCIT 2009. 9th International Symposium on, Sep 2009, pp. 248-253.

[17] W. Song, W. Zhuang, and Y. Cheng, "Load balancing for cellular/WLAN integrated networks," Network, IEEE, vol. 21, no. 1, pp. 27-33, Jan 2007.

[18] D. K. Kim, D. Griffith, and N. Golmie, "A novel ring-based performance analysis for call admission control in wireless networks," Communications Letters, IEEE, vol. 14, no. 4, pp. 324-326, April 2010.

[19] S. Lee, K. Kim, K. Hong, D. Griffith, Y. H. Kim, and N. Golmie, "A probabilistic call admission control algorithm for wlan in heterogeneous wireless environment," IEEE Transactions on Wireless Communications, vol. 8, no. 4, pp. 1672-1676, 2009. 
[20] A. Klein, C. Lottermann, C. Mannweiler, J. Schneider, and H. D. Schotten, "A novel approach for combined joint call admission control and dynamic bandwidth adaptation in heterogeneous wireless networks," in Proc. 7th EURO-NGI Conf. Next Generation Internet (NGI), 2011, pp. $1-8$.

[21] H. Liu, C. Maciocco, V. Kesavan, and A. L. Y. Low, "Energy efficient network selection and seamless handovers in mixed networks," in Proc. IEEE Int. Symp. a World of Wireless, Mobile and Multimedia Networks \& Workshops WoWMoM 2009, 2009, pp. 1-9.

[22] B. Kim, Y. Cho, and J. Hong, "AWNIS: Energy-efficient adaptive wireless network interface selection for industrial mobile devices," Industrial Informatics, IEEE Transactions on, vol. 10, no. 1, pp. 714-729, Feb 2014.

[23] G. Bianchi, "Performance analysis of the ieee 802.11 distributed coordination function," Selected Areas in Communications, IEEE Journal on, vol. 18, no. 3, pp. 535-547, March 2000.

[24] M. Heusse, F. Rousseau, G. Berger-Sabbatel, and A. Duda, "Performance anomaly of 802.11b," in INFOCOM 2003. Twenty-Second Annual Joint Conference of the IEEE Computer and Communications. IEEE Societies, vol. 2, March 2003, pp. 836-843 vol.2.

[25] D.-Y. Yang, T.-J. Lee, K. Jang, J.-B. Chang, and S. Choi, "Performance enhancement of multirate IEEE 802.11 WLANs with geographically scattered stations," Mobile Computing, IEEE Transactions on, vol. 5, no. 7, pp. 906-919, July 2006.

[26] E. Aryafar, A. Keshavarz-Haddad, M. Wang, and M. Chiang, "RAT selection games in HetNets," in INFOCOM, 2013 Proceedings IEEE, April 2013, pp. 998-1006.

[27] G. Auer, V. Giannini, C. Desset, I. Godor, P. Skillermark, M. Olsson, M. Imran, D. Sabella, M. Gonzalez, O. Blume, and A. Fehske, "How much energy is needed to run a wireless network?” Wireless Communications, IEEE, vol. 18, no. 5, pp. 40 -49, Oct 2011.

\section{Appendices}

9.1. Pseudocode of the SOR Algorithm to Calculate the Steady State Probability 


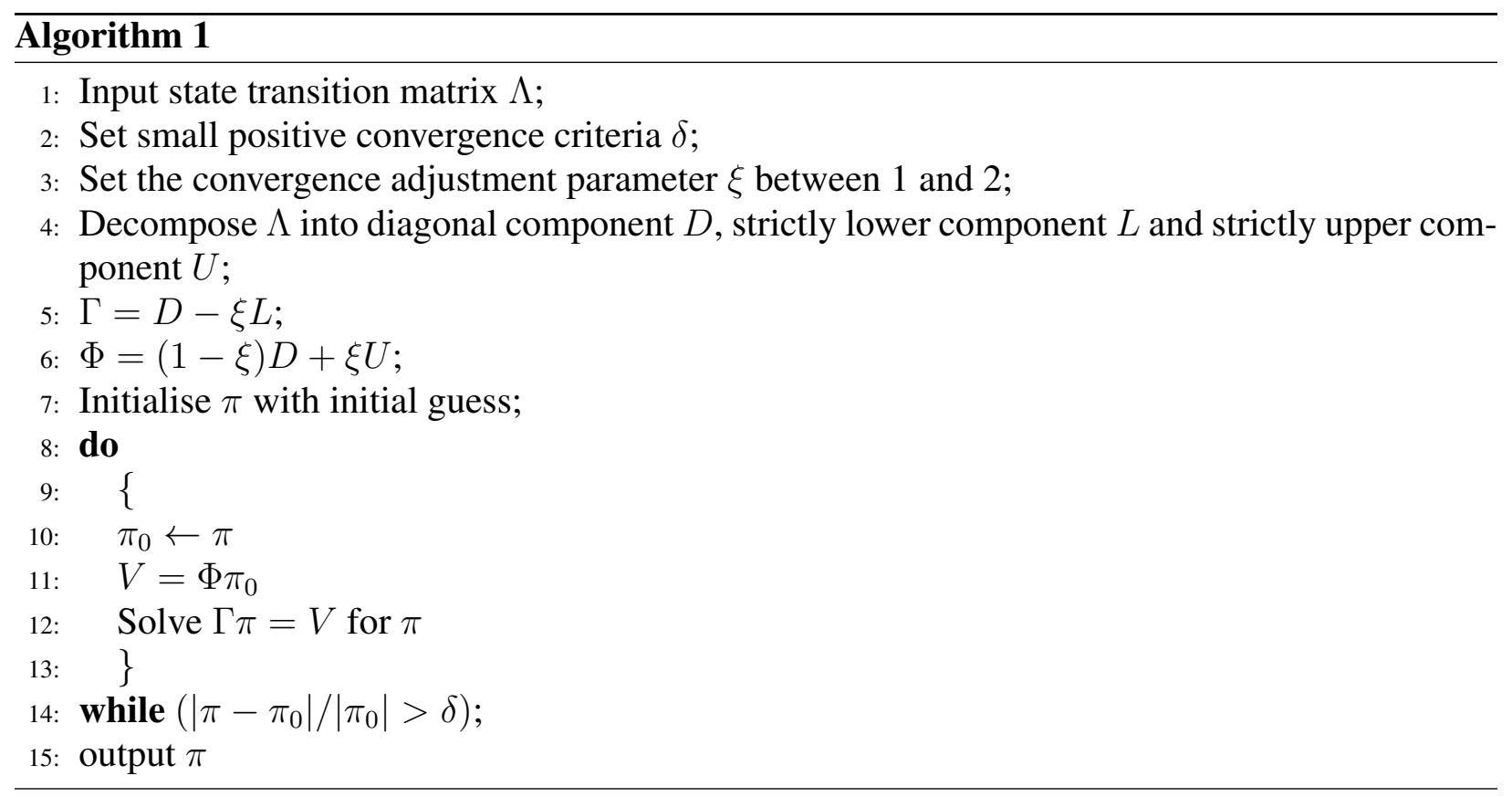

\subsection{Pseudocode of the Exhaustive Search Method for Optimal Preference Values}




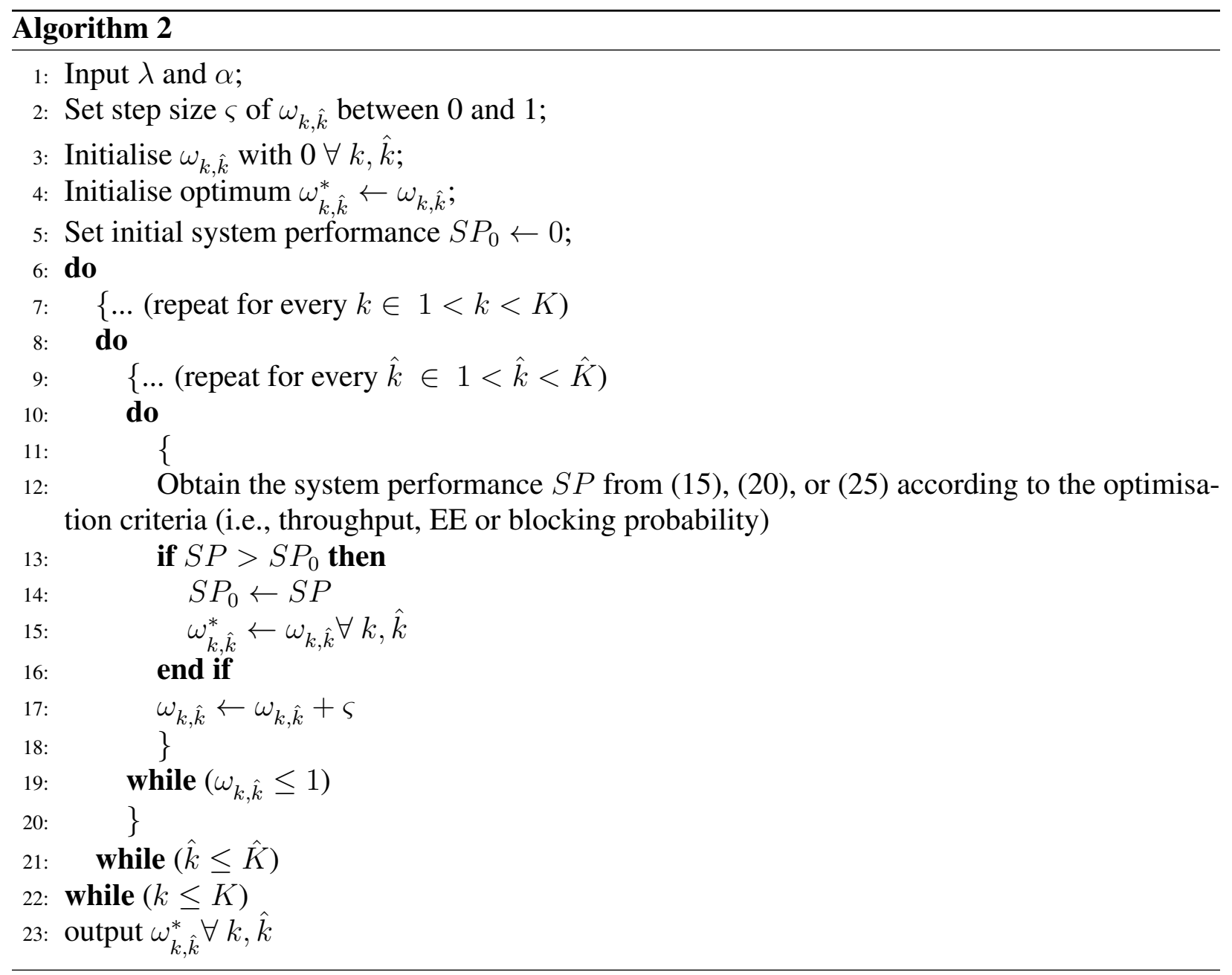

